\title{
Gesetzgebung, verfahrensrechtliche Realität und Rechtslehre im römisch-holländischen Recht des 16. bis 18. Jahrhunderts Das Problem des Mitverschuldens bei Schiffskollisionen
}

This article presents the different solutions provided by various statutes, case law and legal doctrine on the subject of contributory negligence in the event of a collision of ships. With regard to collision cases in Roman-Dutch law, there were various views on the legal solution in cases where two ships collided and both captains had acted negligently. The Hoge Raad decided that where it was not clear who had acted negligently, or where the negligence was equal on both sides, the damage had to be regarded as common and both should bear the damage equally. This decision obviously contrasts with that advanced by Roman law. The Roman approach was adopted as a basis by legal doctrine scholars such as Van Bijnkershoek, and later Van der Keessel, who argued that each should bear his own loss. Interestingly, this view was supported by two local statutes. The article ends with a discussion on another situation, namely that of a stationary ship being hit by a sailing ship. Van der Keessel considered the fault of the sailing ship as more serious and therefore argued that the fault of the (captain of the) sailing ship could not be compensated by the fault of the stationary ship. Thereby he introduced a renewed application of the medieval culpae compensatio doctrine.

\section{Einleitung ${ }^{1}$}

Das Goldene Zeitalter der Niederlande war eine Blütezeit für die Schifffahrt in der Provinz Hol-

\footnotetext{
${ }^{1}$ Dr. E. G. D. van Dongen ist Dozent des Molengraaff Instituts für Privatrecht der Universität Utrecht (Niederlande) und wissenschaftlicher Mitarbeiter am Utrecht Centre for Accountability \& Legal Liability Law (UCALL). Dieser Beitrag basiert auf seinem Vortrag bei dem XVIIIth Annual Forum of Young Legal Historians zum Thema "'Making things legal' Law-making and procedural reality in the European legal traditions" in Wien (Österreich). Dem Beitrag liegt ein überarbeiteter und übersetzter Teil seiner Dissertation zu Grunde, siehe VAN DONGEN, Contributory Negligence, insb. S. 241-255. Diese Untersuchung wird durch die finanzielle Unterstützung der Niederländischen Organisation für wissenschaftliche Forschung (NWO) ermöglicht.
}

land, die zwangsläufig zu einem Anstieg der Zahl der Schiffe führte. Dieser Artikel befasst sich mit Schiffskollisionen und erörtert die unterschiedlichen Lösungsansätze in verschiedenen Gesetzen, in Rechtsprechung und in der Rechtslehre des römisch-holländischen Rechts zur Frage des Mitverschuldens in solchen Fällen. Besonders interessant ist die Frage, inwieweit dabei der römische oder der lokale bzw. regionale Lösungsansatz zugrunde gelegt wurde. Nach der Auslegung des römischen Rechts durch die mittelalterlichen Juristen galt ein Mitverschulden der Geschädigten als anrechenbares Fehlverhalten, das durch die Verweigerung des Anspruchs auf Schadenersatz sanktioniert werden konnte. Hatten zwei Parteien in gleichem Umfang nachlässig gehandelt, wurde beider Nachlässigkeit gegeneinander aufgerechnet. Es 
entwickelte sich die Auffassung, dass die culpa des Schädigers durch die culpa des Geschädigten kompensiert werden könne (culpae compensatio). Dieses „Alles oder nichts“-Prinzip impliziert die Unmöglichkeit einer Schadensteilung. Wurde dieser Ansatz auch im römisch-holländischen Recht zugrunde gelegt? Im vorliegenden Artikel gehe ich zunächst auf die von Grotius beschriebenen Kollisionsfälle ein. Anschließend erörtere ich die drei Situationen, die bei Zusammenstößen allgemein unterschieden werden können: der Schädiger handelte fahrlässig oder vorsätzlich, keine der Parteien handelte schuldhaft (d.h. der Vorfall war die Folge von casus oder es war unklar, wer die Schuld hatte) oder beide Parteien handelten fahrlässig. Den Abschluss bildet meine Schlussfolgerung.

\section{Von Grotius beschriebene Kollisionsfälle}

Hugo de Groot (Grotius, 1583-1645)² beschäftigt sich in seiner Inleidinge mit der damaligen Rechtspraxis in der Provinz Holland. Seine Erörterungen quasi-deliktischer Schiffskollisionen enthalten in Teil 38 zwei relevante Texte zum Mitverschulden in verschiedenen Situationen, in denen die Vorschriften zur Haftung aus unerlaubter Handlung (Aquilian liability) Anwendung fanden. ${ }^{3}$ Der erste Text, Inleidinge III,38,16, beschreibt einen Zusammenstoß zweier Schiffe unter Segeln, der nicht vermieden werden konnte. Wenn ein Schiff sank oder beschädigt wurde, musste der Verlust von beiden Parteien zu gleichen Teilen getragen werden, ungeachtet des Zeitpunkts des Zusammenstoßes (Tag oder Nacht) oder der Wetterbedingungen (gut oder schlecht). Wurde der Zusammenstoß aber durch

\footnotetext{
${ }^{2} \mathrm{Zu}$ Hugo Grotius siehe z.B. EDWARDS, Hugo Grotius; VReElAnd, Hugo Grotius; ZIMMERMANN, Römischholländisches Recht 26ff.; NELLEN, Hugo de Groot.

3 THOMAS, Contributory Fault 347.
}

Vorsatz oder Verschulden einer Partei verursacht, so hatte ausschließlich diese Partei den Schaden zu tragen. ${ }^{4}$ Der zweite Text, Inleidinge III, 38, 18, beschreibt ein Schiff unter Segeln, das ein vor Anker liegendes oder vertäutes Schiff rammt. Hatte dieses Schiff Schaden erlitten, musste das segelnde Schiff für den Gesamtschaden aufkommen, sofern der Zusammenstoß dessen Verschulden war. War dies nicht der Fall, war das segelnde Schiff für die Hälfte des Schadens am anderen Schiff und für seinen eigenen gesamten Schaden haftbar. Die Schuldlosigkeit des segelnden Schiffes musste unter Eid beschworen werden. Konnte das zweite Schiff (das Schiff, das gerammt wurde) jedoch ein Verschulden des segelnden Schiffes beweisen, musste es zudem nachweisen, dass es selbst kein Verschulden daran hatte. ${ }^{5}$ Es gibt aber Situationen, in denen beide Parteien ihr Unverschulden nicht beweisen können. ${ }^{6}$ Die Situation, in der der Geschädigte ebenfalls schuldig war, wird in Grotius' Inleidinge III, 38, 18 nicht beschrieben. Grotius nahm nur bestimmte, aus Statuten ${ }^{7}$ ab-

${ }^{4}$ GROTIUS, Inleidinge III, 38, 16.

${ }^{5}$ GROTIUS, Inleidinge III, 38, 18; THOMAS, Contributory Fault 347f.

${ }^{6}$ In Abschnitt 37 gibt es einen weiteren relevanten Text, und zwar GrotIUS, Inleidinge III, 37, 7, aus dem sich die Anwendung der Regel ableiten lässt, dass die Verantwortlichkeit gleichmäßig verteilt wurde (50/50), falls eine exakte Verteilung aufgrund der Umstände nicht möglich war oder wenn das Verschulden auf beiden Seiten gleich erschien. Diese Regel wurde bereits in den Verfügungen des Hoge Raad angewandt, wie in NeOstadius, Decisiones Nr. 48 und 49 nachzulesen ist.

${ }^{7}$ Nach den Aufzeichnungen von Simon van Groenewegen van der Made zu Grotius' Inleidinge (die Ausgabe des Jahres 1692, i.c. 306, wird verwendet; siehe auch THOMAS, Contributory Fault 348), muss Grotius die Ordonnanz von Kaiser Karl V. zu seerechtlichen Angelegenheiten aus dem Jahr 1551, die Ordonnanz von König Philipp von Spanien zum selben Thema aus dem Jahr 1563 sowie die Gesetze von Wisby verwendet haben. Infolge einer redaktionellen Überarbeitung enthält die Veröffentlichung „Gesetze von Wisby“ faktisch zwei 
geleitete Regeln in das System deliktischer und quasi-deliktischer Schadenersatzansprüche auf. Er äußerte sich nicht zu der Möglichkeit, dass beide Schiffe Schuld am Zusammenstoß haben. Dennoch bildeten diese Texte die Grundlage für die Entwicklung des Konzepts des Mitverschuldens im römisch-holländischen Recht. ${ }^{8}$

\section{Schiffskollisionen, bei denen die alleinige Schuld bei einem der Schiffe lag}

In Fällen, in denen der Schiffsschaden durch Verschulden eines der beiden Schiffe oder beider Schiffe verursacht wurde, bildete römisches Recht den Ausgangspunkt. Da es aber keine spezifische römische Rechtsvorschrift $\mathrm{zu} \mathrm{Zu}$ sammenstößen gab, fanden die allgemeinen Vorschriften Anwendung. Bei Schiffskollisionen galt die lex Aquilia. ${ }^{9}$ Die Haftung der Personen, die das Schiff navigierten, basierte auf der lex Aquilia und genauer auf der Frage, ob ihnen culpa zuzurechnen war oder ob sie dolus zu vertreten hatten. ${ }^{10}$ Darüber hinaus konnte im Falle

andere Sammlungen, und zwar die Urteile zum Seerecht aus Damme in Flandern (eine flämische Umsetzung einer Sammlung von Urteilen des Seegerichtshofs der französischen Insel Oléron) sowie die gesammelten Handelsbräuche in seerechtlichen Angelegenheiten in Amsterdam (die nach THOMAs, Contributory Fault 350, wahrscheinlich aber aus Staveren stammten). Siehe VERWER, Nederlants seerechten, besonders VIIIff.

8 THOMAs, Contributory Fault 351.

${ }^{9}$ Siehe insbesondere D 9, 2, 29, 2 und 4; siehe auch PÖHLS, Handelsrecht III, 778; LOBINGIER, Maritime Law $29 f$.

${ }^{10}$ Siehe AsHbURner, Nomos Rodion Nautikos cclxxxv; GoRMLEY, Rhodian-Roman Maritime Law 327; Der Schiffseigner (exercitor) ist möglicherweise für Waren und sonstiges Eigentum, deren sicheren Transport er übernommen hat, für Schäden/Verletzungen, die von der Mannschaft an Bord des Schiffs den Waren oder Personen zugefügt werden und für vom Magister eines unbeabsichtigten Verlustes nach den in D 9, 2, 29, 2-5 beschriebenen römischen Rechtsvorschriften vom Eigentümer des geschädigten Schiffes keine auf der lex Aquilia beruhende Klage erhoben werden. Cornelis van Bijnkershoek (1673-1743) $)^{11}$ gründete seine Meinung auf römisches Recht, als er darlegte, der Schaden sei von der Partei, die nachlässig gehandelt habe, zu tragen. Er beschrieb das Beispiel eines Schiffes unter Segeln, das ein anderes Schiff unter Segeln, ein anderes verankertes oder vertäutes Schiff oder aber ein treibendes Schiff beschädigte, das den gesamten Schaden zu tragen habe, falls der Schaden durch eigene Verschulden verursacht wurde. ${ }^{12}$

\section{Schaden infolge von Unfall}

Nach mittelalterlicher Auslegung des römischen Rechts konnte nicht gegen den Schädiger vorgegangen werden (sofern die Handlung nicht gesetzlich verboten war), wenn der Schaden durch einen Unfall verursacht wurde. D 9, 2, 29 enthält keine explizite Rechtsvorschrift bezüglich einer Situation, in der keine Nachlässigkeit einer der betroffenen Parteien nachgewiesen werden kann. ${ }^{13}$ In seinem De iure belli ac pacis legte Grotius dar, dass das Naturrecht vorschreibt, dass eine Person, deren Schiff dem Schiff eines anderen ohne Verschulden Schaden zufügt, nicht

erstellte Verträge haftbar. Siehe AsHBURNER, Nomos Rodion Nautikos clix-clxiii.

${ }^{11} \mathrm{Zu}$ Van Bynkershoek siehe z.B. STAR NumAN, Cornelis van Bynkershoek; ZIMMERMANN, Römischholländisches Recht 32ff.; VAN DEN BERGH, Präsident 423ff.; BROM, Urteilsbegründungen 27ff.; VAN BINNERSHOEK, Quaestionum iuris privati libri quatuor (1744) wurde 1747 ins Niederländische übersetzt und war ein Handbuch, das in der Rechtspraxis vielfach Anwendung fand. Siehe VAN DEN BERGH, Präsident 432.

12 VAN BIJNKERSHOEK, Quaestiones juris privati IV, 22; THOMAS, Contributory Fault 355f.

${ }^{13}$ Siehe auch Lichtenauer, Geschiedenis 192. 
haftbar ist (diese Sichtweise würde dem römischen Recht entsprechen). Nach dem Recht vieler Völker sowie nach dem Recht der Provinz Holland, fährt Grotius fort, würde ein solcher Schaden angesichts der Problematik der Klärung der Schuldfrage im Allgemeinen jedoch zwischen beiden Parteien verteilt. ${ }^{14}$

Das Prinzip, dass im Falle eines Zusammenstoßes zweier Schiffe, bei dem keiner der Parteien Verschulden nachgewiesen werden kann, der Schaden zwischen ihnen verteilt wurde, ist eine Grundregel, die im Schifffahrtsrecht bereits seit dem Mittelalter angewandt wird und die im altdeutschen Gedanken der genossenschaftlichen Schadensgemeinschaft begründet ist. ${ }^{15}$ Nach dem Seerecht musste der Schaden im Falle eines Unfalls, bei dem keine der Parteien fahrlässig handelte, dennoch zum Teil vergütet werden. ${ }^{16}$ Bei Schaden durch einen Zusammenstoß von Schiffen ohne (bewiesenermaßen vorhandenes) Verschulden vonseiten einer der Parteien, wurde der Schaden von beiden Parteien zu gleichen Teilen (50/50) getragen. ${ }^{17}$ Diese Regel fand im römisch-holländischen Recht des siebzehnten und achtzehnten Jahrhunderts praktische Anwendung. ${ }^{18}$

${ }^{14}$ GrotiUs, De iure belli ac pacis II, 17, 21. Siehe auch AUMANN, Mitwerkende Verschulden 158; PARISI, Liability 146; DERS., Alterum non laedere 341, Anm. 74

${ }^{15}$ AUMANN, Mitwerkende Verschulden 157f.; LUIG, Überwiegendes Mitverschulden 225.

${ }^{16}$ Siehe VAN DER KEESSEL, Praelectiones ad III, 37, 7.

${ }^{17}$ Siehe auch VAN BIJNKERSHOEK, Quaestiones juris privati IV, 18; Diese Regel (rusticum judicium or judicium rusticorum, die von Schiedsgerichten verfügte Teilersatz der Hälfte des Schadens) findet man bereits in Art. 14 Rôles d'Oléron, Art. 48, 49, 50 \& 71 der Wisbuische Seerechten, Art. 49 des Placaet ende Ordonnantie, Op 't stuck vande Zee-Rechten vom 19.7. 1551, Art. 4 des Titels 'Van Schepen die malkanderen beschadigen' der Ordonnantie, Statuyt ende eeuwige Edict etc. vom 31.10. 1563. Siehe auch LICHTENAUER, Geschiedenis 192.

${ }^{18}$ Siehe NeOstadius, Decisio Nr. 48; VAN BIJNKERSHOEK, Observationes Tumultuariae 1320.
Diese Regel fand in dem Fall Anwendung19, über den das Gericht erster Instanz, in Berufung und letztendlich in der Revision am 12. Februar 1717 vom Ho(o)ge Raad van Holland, Zeeland en West-Friesland ${ }^{20}$ entschied. Ein aus Hamburg kommendes Schiff erreichte die Insel Texel und verließ die Insel dann zusammen mit anderen Schiffen im Konvoi des Admirals von Holland. In einem gewissen Moment befahl der Admiral dem Konvoi einen Kurswechsel. Das Schiff aus Hamburg folgte dem Befehl, aber ein anderes Schiff, das zur niederländischen Ostindischen Kompanie gehörte, konnte dieses Manöver aufgrund seiner schweren Ladung und der relativ rauen See nicht durchführen und kollidierte mit dem Schiff aus Hamburg. Infolge dieser Havarie sank das Hamburger Schiff. Die Eigentümer des Hamburger Schiffes machten die Hälfte des Schadens bei der niederländischen Ostindischen Kompanie geltend. ${ }^{21}$ In diesem Fall wurde erwogen, dass der Unfall durch Zufall und nicht infolge von Verschulden einer der Parteien entstanden sei. Der Hoge Raad entschied daher, dass der Schaden von beiden Parteien getragen werden müsse. ${ }^{22}$

Die Methode, derzufolge das Gericht zu seiner Einschätzung gelangte, war Gegenstand von Diskussionen. Während die vorherrschende Meinung der Richter des Hoge Raad am 23. November 1720 die Auffassung vertraten, dass eine solche Beurteilung mittels arithmetischer Be-

${ }^{19}$ Diese Regel fand auch in einer Entscheidung des Hof van Holland vom 4.7.1662 Anwendung, aufgezeichnet von P. Ockers (1628-1678), siehe OCKERS, Decisien, Teil 1, Titel 3, Dez. 1.

${ }^{20}$ Jüngst, im Rechtsprechung des Ho(o)ge Raad van Holland, Zeeland en West-Friesland, siehe PUNT, Vennootschapsrecht 3ff., der ebenfalls Themen wie die Zuständigkeiten und die Zusammensetzung des Gerichts usw. behandelt.

${ }^{21}$ Siehe VAN BIJNKERSHOEK, Observationes Tumultuariae 1320.

${ }^{22}$ Ebd., Van Bijnkershoek referierte an Art. 50, 70 und 73 der Wisbuische Seerechten; dieser Fall wurde von WESKETT, Complete Digest 479f., besprochen. 
rechnung zu erfolgen habe, ${ }^{23}$ vertrat Van Bijnkershoek in seinen Quaestiones juris privati die Meinung, dass die Parteien in Fällen ohne bewiesene Schuld einer der Parteien einen Verlust im Verhältnis zum Wert des Schiffes und der Ladung tragen sollten, d.h. nach Maßgabe einer geometrischen Berechnung. ${ }^{24}$ Er führte an, dass in einer Situation, in der beide Parteien durch die Kollision Schaden erlitten, dieser Schaden aber nicht durch Nachlässigkeit einer der Parteien verursacht wurde, beide Parteien den Schaden gleichmäßig im Verhältnis zu den Mitteln oder dem Wert des Eigentums der jeweiligen Partei tragen sollten. ${ }^{25}$ Die Hälfte des Wertes des

${ }^{23}$ Ebd., und siehe auch VAN BIJNKERSHOEK, Observationes Tumultuariae 1711 [686]; BOUMAN, Dissertatio 63; Van Bijnkershoek referiert an die Entscheidungen des Hohe Rat von Holland, Seeland und Westfriesland, aufgezeichnet in NeOsTADIUS, Decisiones 48 und 49 [ohne Datumsangabe] und die Entscheidungen vom 21.12.1629 (COOREN, Observatio 40) und 17.5.1630 (COOREN, Observatio 41); Spätere holländische Gelehrte waren unschlüssig $\mathrm{zu}$ diesem Thema. Siehe $\mathrm{zu}$ diesem Thema auch TAunAY, Disputatio 42ff. Van der Keessel und Van der Linden erwähnten beide Sichtweisen (die geometrische und die arithmetische Berechnung), siehe VAN DER KEESSEL, Theses selectae, These 815; VAN DER LINDEN, Koopmanshandboek IV, 5, 7.

${ }^{24}$ VAN BIJNKERSHOEK, Quaestiones juris privati IV, 20.

${ }^{25}$ Ein mittelalterlicher Grundsatz des Seerechts lautete, ein Schiffseigner könne auf einer Fahrt nicht mehr verlieren, als ihm anvertraut wurde oder er dabei riskiert habe, siehe S'JACOB, Recht van abandon 14, 32; VAN NIEKERK, Principles of Insurance Law 1282. Wenn der Kapitän eines Schiffes Schaden verursachte, während er seinem Aufgabenbereich entsprechend handelte, war die Haftung des Schiffseigners somit nur auf den Wert seines Schiffes begrenzt. Nach Aussage von VAN NIEKERK, Principles of Insurance Law 1282f., konnte Rechtsprechung gegen den Schiffseigner nur gegen das Schiff erhoben werden, das zu diesem Zweck beschlagnahmt wurde. Bei Überlassung oder Abtretung (oder: fiktiver Abtretung) des Schiffes $\mathrm{zu}$ diesem Zweck und Nichterscheinen zur persönlichen Verteidigung im Verfahren beschränkte/n der/die Schiffseigner faktisch seine/ihre Haftung auf (ihren Anteil an dem) den Wert des Schiffes. Die Haftungsbeschränkung gesunkenen Schiffes wird jedoch den Gesamtwert des verbleibenden Schiffes absolut nicht überschreiten, da der Betrag, den das gesunkene Schiff mehr wert ist, vom geschätzten Gesamtschaden abgezogen wird. ${ }^{26}$ Dies stimmt mit einem Urteil des Hoge Raad aus dem Jahr 1629 überein, demnach es absurd sei, dass die Eigentümer für mehr als die Hälfte des Wertes des verbleibenden Schiffes haftbar seien, da sie in diesem Falle ja mehr zahlen würden als das, was für sie selbst auf dem Spiel stand. ${ }^{27}$ Auf diese Weise, d.h. aus der Sicht von Van Bijnkershoek, wird die Haftung des Schiffeigentümers auf den Wert seines eigenen Schiffes beschränkt. Die Eigentümer werden von weiteren Ansprüchen befreit, indem sie das aufgeben, was ihnen gehörte. Die Regel der gleichmäßigen Verteilung war vordem nicht allgemein anwendbar; die Haftung für die Hälfte des Schadens am anderen Schiff konnte nicht mehr über den (unbeschädigten) Wert des eigenen Schiffes des Eigentümers hinausgehen. ${ }^{28}$

\section{Kollisionsschaden durch Verschulden beider Parteien}

Nach römisch-holländischem Recht hatte eine Person, die rechtswidrig und schuldhaft Scha-

des Schiffseigners - auch im römisch-holländischen Recht anerkannt - wurde durch eine Personifizierung seines Schiffes bewirkt: das Schiff wurde gewissermaßen als haftbar für die Handlungen seines Kapitäns bei der Fortsetzung ihrer Reise angesehen. Da es aus der Natur der Sache allerdings unmöglich war, ein Schiff zu verklagen, wurde die Klage daher an den/die Schiffseigener gerichtet. Siehe MuNNIKS, Handleiding 47; VAN NIEKERK, Principles of Insurance Law 1282; zum Recht der Überlassung im römischholländischen Recht siehe S'JACOB, Recht van abandon 26ff.

${ }^{26}$ VAN BIJNKERSHOEK, Quaestiones juris privati IV, 20.

${ }^{27}$ Siehe COOREN, Observatio 40, Nr. 26.

${ }^{28}$ Siehe auch Magens, Essay 78; WesketT, Complete Digest 481; VAN NIEKERK, Principles of Insurance Law 1295, Anm. 53. 
den zufügte, für den Verlust aufzukommen. Aber was geschieht, wenn beide an der Kollision beteiligten Parteien nachlässig handelten? ${ }^{29} \mathrm{Ob}$ wohl argumentiert werden könnte, dass in diesem Fall beide Parteien für ihren jeweiligen Schaden aufkommen sollten, lässt sich diese Möglichkeit in Kollisionsfällen nicht finden. ${ }^{30} \mathrm{Im}$ römisch-holländischen Recht findet man jedoch zwei unterschiedliche Sichtweisen: die Auffassung des Hoge Raad und die Auffassung, die von Van Bijnkershoek vertreten wurde.

In einem vom Hoge Raad behandelten Fall, der von Cornelis van Nieustadt (Neostadius, 15491606) $)^{31}$ als decisio 49 aufgezeichnet wurde, wird das Mitverschulden des Geschädigten vom Hof van Holland und dem Hoge Raad ausdrücklich erwähnt. In diesem Fall ging es um eine Kollision zweier Schiffe, die das Sinken eines der Schiffe zur Folge hatte. Der Schiffer des gesunkenen Schiffes verklagte den Eigentümer des anderen Schiffes. Der Angeklagte entgegnete jedoch, dass die Kollision nicht auf einen Fehler seinerseits zurückzuführen sei. Im Gegenteil, der Unfall sei durch den Kapitän des gesunkenen Schiffes verursacht worden, der am helllichten Tage, während die Gäste aßen, einem unerfahrenen Seekadetten das Steuerruder überlassen habe. Die Unerfahrenheit dieses Mannes sei die Ursache für die Kollision der Schiffe gewesen. ${ }^{32}$ Der Hof van Holland und der Hoge Raad gelangten zu

\footnotetext{
${ }^{29}$ Eine kurze Zusammenfassung wird von KOTZÉ, Aanspreeklikheid 67f., geboten.

${ }^{30}$ Simon van Leeuwen war der Auffassung, bei einem Zusammenstoß von Wagenfahrern mit Nachlässigkeit von beiden Seiten müssten die Parteien für ihren eigenen Schaden aufkommen; siehe VAN LEEUWEN, Rooms-Hollands regt IV, 39, 8 .

${ }^{31} \mathrm{Zu}$ Neostadius siehe z.B. AHSMANN, Collegia en colleges 46ff. Die Sammlung mit dem Titel 'Utriusque Hollandiae, Zelandiae, Frisiaeque Curiae Decisiones' (erste Ausgabe 1617) war fälschlicherweise Neostadius zugeschrieben worden. $\mathrm{Zu}$ diesem Thema siehe VAN APELDOORN, Handschrift 3ff.; ZEYLEMAKER, Geschiedenis 123f.; AHSMANN, Collegia en colleges 48. ${ }^{32}$ NeOstadius, Decisio Nr. 49.
}

dem Schluss, dass der Schaden von beiden Parteien zu gleichen Teilen zu tragen sei, da beide Seiten gleich viel Schuld hätten. ${ }^{33}$

In Bezug auf diesen Fall wählte Van Bijnkershoek jedoch den römischen Ansatz und argumentierte, dass die Schlussfolgerung, dass keine der Parteien Klage erheben könne und somit jedes Schiff für den eigenen Schaden aufkommen müsse, zutreffender sei. Wer nachlässig handle, könne keine Klage erheben. ${ }^{34}$ Der Kläger erlitt Schaden, der jedoch durch sein eigenes Verschulden verursacht wurde; cf. D 50, 17, 203 galt somit, dass er keinen Schaden erlitten habe. Zudem würde es einem Beklagten nicht helfen, wenn er vorgäbe, die andere Partei habe ebenfalls nachlässig gehandelt. Um Klage erheben zu können, musste er das Gesetz eher auf seiner Seite haben. Eine Person, die nachlässig handelte, konnte keine Klage erheben. Wenn beide Parteien nachlässig handelten, mussten sie jeweils ihren eigenen Schaden tragen. ${ }^{35}$

Gegen seine eigene Auffassung führte Van Bijnkershoek jedoch ein Gegenargument an, nämlich, dass mit Art. 3, Abs. 5 der Ordonnanz von König Philipp ${ }^{36}$ eine Ausnahme vom römischen Recht eingeführt sein könne. Wenn beide Parteien einer Kollision schuldig seien, wären keine Rechtsmittel verfügbar und müsse jede der Parteien den eigenen Verlust tragen. Nach diesem Artikel hat ein Schiff unter Segeln, das mit einem vor Anker liegenden Schiff zusammenstößt, seinen eigenen Verlust zu tragen. Der Schaden am ruhenden Schiff war zu gleichen Teilen $\mathrm{zu}$ tragen, davon ausgehend, dass die Besatzung des Schiffs unter Segeln unter Eid erklärt hat, keinerlei Schuld zu tragen. Konnte

\footnotetext{
33 Ebd.; PÖHLs, Handelsrecht III, 778; BOUMAN, Dissertatio 42; THOMAS, Contributory Fault 355.

${ }^{34}$ Siehe auch KotzÉ, Aanspreeklikheid 68.

${ }^{35}$ VAN BIJNKERSHOEK, Quaestiones juris privati IV, 22; KOTZÉ, Aanspreeklikheid 68. Entsprechend VAN DER LINDEN, Adnotatio ad Voet D 9, 2, Nr. 15.

${ }^{36}$ Siehe CAU, Groot placaet-boeck, I, 817.
} 
das ruhende Schiff jedoch beweisen, dass das segelnde Schiff Schuld hatte, war dieses für den Gesamtverlust haftbar, sofern das ruhende Schiff nachweisen konnte, dass es selbst keine Schuld trug. ${ }^{37}$ Van Bijnkershoek warf die Frage auf, ob die Regel der gleichmäßigen Verteilung des Schadens im Fall des ruhenden Schiffes zuträfe, wenn es gelänge, die Schuld des Schiffs unter Segeln zu beweisen, die absolute Schuldfreiheit auf der eigenen Seite nicht unter Beweis gestellt werden konnte. Van Bijnkershoek lehnte diese Lösung ab und erklärte, die korrekte Auslegung sei die, dass die schuldige Partei alle Schäden zu tragen habe, sofern der Geschädigte nicht ebenfalls schuldig sei. Im letzteren Fall hätten beide Seiten ihre eigenen Verluste zu tragen. ${ }^{38}$ Diese Lösung wurde von Dionysius Godefridus van der Keessel (1738-1816) übernommen. ${ }^{39}$

Interessanterweise interpretierte Van der Keessel Art. 3, Abs. 5 der Ordonnanz von König Philipp im Zusammenhang mit einem Fall des Mitverschuldens jedoch dahingehend, dass das Verschulden eines Schiffes unter Segeln im Falle eines Zusammenstoßes schwerer wiege als die eines ruhenden Schiffes. Laut Van der Keessel könne die Schuld des (Kapitäns des) segelnden Schiffes nicht durch die Schuld des ruhenden

\footnotetext{
${ }^{37}$ Siehe auch THOMAS, Contributory Fault 356.

${ }^{38}$ Ebd.; Van Bijnkershoek fand Unterstützung für seine Argumente in Art. 46 des Placaet von Charles und in Art. 1 des Titel 5 des Placaet von Philipp, das angab, dass nur im Falle beiderseitiger Schuldfreiheit der Schaden von beiden Parteien zu gleichen Teilen getragen werde.

${ }^{39}$ Siehe VAN BIJNKERSHOEK, Quaestiones juris privati IV, 22. VAN DER KEESSEL, Theses selectae, Thesis 816. Diese von Van der Keessel ergriffene Lösung wurde ebenfalls von zwei lokalen Statuten unterstützt, und zwar durch Art. 262 Ordonnantie von Rotterdam aus dem Jahr 1721 und Art. 179 Instructie von Dordrecht aus dem Jahr 1775; Zu Van der Keessel siehe z.B. KRIKKE, FABER, Dionysius Godefridus van der Keessel 185ff.; FeENSTRA, Keessel, Dionysius Godefridus van der 353.
}

Schiffes aufgehoben werden, und das Schiff unter Segeln müsse die Hälfte des Verlustes des vor Anker liegenden Schiffes tragen. ${ }^{40}$ Daraus lässt sich folgern, dass er das Verschulden beider Parteien quantitativ bewertete. Er führte eine neue Anwendung der vorab erwähnten mittelalterlichen culpae compensatio-Lehre ein. Eine vergleichbare Theorie kam bereits durch Johannes Voet (1647-1713) ${ }^{41}$ in seiner Erörterung von D 9, 2, 11, pr. zur Anwendung, indem er durch Quantifizierung der jeweiligen Schuld und durch die Aussage, der Schädiger sei nur dann für den gesamten Verlust haftbar, wenn seine Schuld größer war, eine Ausnahme der römischen Regel erstellte. ${ }^{42}$ Im Gegensatz zu Voets Weise der Anwendung dieser Einschätzung war die Partei mit der größeren Nachlässigkeit bei Van der Keessel nicht für den Gesamtschaden haftbar, sondern musste neben dem eigenen Schaden nur die Hälfte des Schadens der Gegenpartei tragen. ${ }^{43}$

Außerdem argumentierte Van der Keessel, bei einem Zusammenstoß zwischen einem Schiff unter Segeln und einem vor Anker liegenden Schiff sei Nachlässigkeit von Seiten des Kapitäns

\footnotetext{
${ }^{40}$ Siehe VAN DER KeESSEL, Praelectiones ad III, 38, 16; THOMAs, Contributory Fault 357.

${ }^{41} \mathrm{Zu}$ Johannes Voet siehe z.B. KoP, Johannes Voet 130ff.; ZIMMERMANN, Römisch-holländisches Recht 39ff.; ASHMANN, Voet, Johannes 656.

${ }^{42}$ Voet, Commentarius ad D 9, 2, Nr. 17.

${ }^{43}$ Siehe auch THOMAS, Contributory Fault 358f.; Letztendlich führte VAN DER LINDEN, Koopmanshandboek IV, 5, 7 an, dass bei gleicher Schuld beider Seiten beide Seiten den eigenen Verlust zu tragen haben. War die Schuldfrage der Kollision unklar, trug jedes Schiff die Hälfte des Schadens. Leider ging Van der Linden nicht auf die Frage oder das Ergebnis einer größeren Schuld einer der Parteien ein. Laut THOMAS, Contributory Fault 358 umging Van der Linden das Thema des Mitverschuldens bei ungleicher Nachlässigkeit. Thomas bemerkte $\mathrm{zu}$ Recht, dass Van der Lindens Weise der Formulierung allgemeiner Regeln den besonderen Fall eines segelnden Schiffs, das ein vertäutes oder verankertes Schiff rammt, nicht außer Acht lassen kann.
} 
oder der Besatzung vorausgesetzt, da ein Schiff normalerweise über ein Steuerruder gelenkt würde. Van der Keessel zufolge lautete die richtige Auslegung von Art. 3 des vorab erwähnten Erlasses von König Philipp, die Haftung eines Schiffes unter Segeln sei auf die Hälfte des Gesamtschadens des ruhenden Schiffes begrenzt, wenn dem vor Anker liegenden Schiff Nachlässigkeit in irgendeiner Form vorzuwerfen sei. ${ }^{44}$ Der Erlass von König Philipp wurde durch Art. 262 der Ordonnantie van Rotterdam angepasst, der die Regel enthielt, dass ein Schiff, das gerammt wurde, den Schaden zu tragen habe, wenn es die Kollision hätte vermeiden können, da der Schaden auf Nachlässigkeit des Kapitäns zurückzuführen sei. Auf diese Weise wurde der römische Grundsatz D 50, 17, 203 durch das Gesetzesrecht wieder eingeführt. ${ }^{45}$

\section{Schlussfolgerung}

Der Hoge Raad entschied, ein Schaden sei als gemeinsam anzusehen und von beiden Parteien $\mathrm{zu}$ gleichen Teilen zu tragen, wenn bei einem Zusammenstoß nicht deutlich feststehe, welcher Kapitän nachlässig gehandelt habe oder wenn an beiden Seiten von Verschulden die Rede sei. Diese Entscheidung widerspricht augenscheinlich den Regeln des römischen Rechts und den Regeln in ius commune. Der Ansatz des Hoge Raad beruhte jedoch auf dem Seerecht (dem Konzept der Schadensgemeinschaft). Einige Rechtsgelehrte wichen jedoch von dieser im achtzehnten Jahrhundert herrschenden Sichtweise ab. Sie übernahmen den römischen Ansatz (und wandten die Regel aus D 50, 17, 203 an) mit der Begründung, dass jedes Schiff seinen eigenen Schaden zu tragen habe, wenn beide

\footnotetext{
${ }^{44}$ VAN DER KEESSEL, Praelectiones ad III, 38, 18; DERS., Theses selectae, Thesis 821; THOMAS, Contributory Fault 357f.

${ }^{45}$ Entsprechend THOMAS, Contributory Fault 358.
}

Seiten fahrlässig waren. Interessanterweise wurde diese Sichtweise durch die Stadtrechte der Städte Rotterdam und Dordrecht gestützt.

Im Falle eines ruhenden Schiffes, das von einem Schiff unter Segeln gerammt wurde, argumentierte Van der Keessel, dass die Schuld des (Kapitäns des) segelnden Schiffes nicht durch die Schuld des ruhenden Schiffes aufgehoben werden könne und dass das Schiff unter Segeln die Hälfte des Schadens des vor Anker liegenden Schiffes zu tragen habe. Entgegen Voets Weise der Anwendung dieser Einschätzung - die sich aus der Erörterung von D 9, 2, 11, pr. ergibt - sei die Partei mit dem größeren Verschulden nicht für den Gesamtschaden haftbar, sondern habe neben dem eigenen Schaden lediglich die Hälfte des Schadens der Gegenpartei zu tragen. Dieser Unterscheid lässt sich durch die Tatsache erklären, dass das Seerecht im Falle eines durch casus verursachten Zusammenstoßes vorsah, dass die Hälfte des Schadens kompensiert werden müsse (während das römische Recht hier keine Haftung anführen würde). Nach Van der Keessel fiel man auf das casus-System zurück, sofern das Mitverschulden der Geschädigten erheblich geringer als das Verschulden des Schädigers war und man nur die Hälfte des Schadens geltend machen konnte.

\section{Korrespondenz:}

E.G.D. van Dongen

Molengraaff Instituut voor Privaatrecht I Utrecht Centre for Accountability \& Legal Liability Law (UCALL) Universiteit Utrecht Janskerkhof 12, 3512 Utrecht, Belgium E.G.D.vanDongen@uu.nl 


\section{Abkürzungen:}

RIDA Revue internationale des droits de l'antiquite

ZEuP Zeitschrift für europäisches Privatrecht

\section{Literatur:}

Walter ASHBURNER, Nomos Rodion Nautikos: The Rhodian Sea-Law (Oxford 1909, ND Aalen 1976).

Margaretha J. A. M. AHSMANN, Collegia en colleges. Juridisch onderwijs aan de Leidse Universiteit 1575-1630, in het bijzonder het disputeren (Groningen 1990).

DIES., Voet, Johannes (1647-1713), in: Michael STOLLEIS (Hg.), Juristen. Ein biographisches Lexikon. Von der Antike bis zum 20. Jahrhundert (München 22001) 656.

Peter AumAnN, Das mitwirkende Verschulden in der neueren juristischen Dogmengeschichte (Hamburg 1964).

Lodewijk J. H. BOUMAN, Dissertatio juridica inauguralis de collisione navium (Rotterdam 1855).

Christian BROM, Urteilsbegründungen im «Hoge Raad van Holland, Zeeland en West-Friesland » am Beispiel des Kaufsrechts im Zeitraum 17041787 (= Rechtshistorische Reihe 377, Frankfurt am Main 2008).

Cornelis CAU (Hg.), Groot placaet-boeck, 2 Bde. (The Hague 1658/1664).

Jacobus COOREN, Observationes rerum in eodem senatu iudicatarum (Amsterdam 1661).

Charles S. EDWARDS, Hugo Grotius: The Miracle of Holland (Chicago 1981).

R. FeEnStRA, Keessel, Dionysius Godefridus van der (1738-1816), in: Michael STOLLEIS (Hg.), Juristen. Ein biographisches Lexikon, von der Antike bis zum 20. Jahrhundert (München 22001), 353.

Helene C. GALl (Hg.), Regtsgeleerde decisien. Aan de raadsheer Pieter Ockers toegeschreven aantekeningen betreffende uitspraken van het Hof (1656-1669) en de Hoge Raad (1669-1678) van Holland, Zeeland en West-Friesland (Amsterdam 2002).

W. P. GORMLEY, The Development of Rhodian-Roman Maritime Law to 1681, with Special Emphasis on the Problem of Collision, in: Inter-American Law Review 3 (1961) 317-345.

Hugo Grotius, De iure belli ac pacis libri tres, hrsg. von B. J. A. DE KANTER-VAN Hettinga Tromp (Leiden 1939, ergänzter ND Aalen 1993).
DERS., Inleidinge tot de Hollandsche rechtsgeleerdheid, hrsg. von F. DovRING, H. F. W. D. FISCHER, E.M. MEIJERS (Leiden 1952).

DERS., Inleydinge tot de Hollandse regts-geleertheyt, mitsgaders eenige byvoegsels en aanmerkingen op de selve, door S. van Groenewegen van der Made (Amsterdam 1692).

Instructie voor schepenen van 't watergerecht, en ordonnantie op het stuk van assurantie en avarye, mitsgaders van zeezaken en de manier van procederen ontrent dezelve (Dordrecht 1775).

Charles M. S'JАСОВ, Het recht van abandon (The Hague 1890).

Peter C. KoP, Johannes Voet (1647-1713), in: Theo J. VeEN, Peter C. KoP (Hgg.), Zestig juristen. Bijdragen tot ene beeld van de geschiedenis der Nederlandse rechtswetenschap, m.m.v. C.H.N. KWANTEN (Zwolle 1987) 130-134.

Petrus J. KoTZÉ, Die aanspreeklikheid van mededaders en afsonderlike daders (Leiden 1953).

A. KRIKKE, S. FABER, Dionysius Godefridus van der Keessel (1738-1816), in: Theo J. VEEN, Peter KOP (Hgg.), Zestig juristen. Bijdragen tot ene beeld van de geschiedenis der Nederlandse rechtswetenschap (Zwolle 1987) 185-189.

Charles S. LOBINGIER, The Maritime Law of Rome, in: Juridical Review 47 (1935) 1-32.

Wilhelm F. LICHTENAUER, Geschiedenis van de wetenschap van het handelsrecht in Nederland tot 1809 (Amsterdam 1956).

Klaus LUIG, Überwiegendes Mitverschulden, in: Ius Commune 2 (1969) 187-238.

Nicolas MAgeNS, An Essay on Insurances, Bd. I (London 1755).

Johannes MUNNIKS, Handleiding tot de hedendaagsche rechtsgeleerdheid der Vereenigde Nederlanden, naar den order van het Romeinsche recht (Amsterdam 1776).

Henricus J. M. NelLEN, Hugo de Groot. Een leven in strijd om de vrede 1583-1645 (Amsterdam 2007).

Ordonnantie op het stuk van asseurantie ende van avarye: mitsgaders zee-zaken (1721, ND Rotterdam 1748).

Francesco PARISI, Liability for Negligence and Judicial Discretion, with foreword by P. STEIN (Berkeley 21992).

DERS., Alterum non laedere. An Intellectual History of Civil Liability, in: Am. J. Juris. 39 (1994) 317-551.

Meno PÖHLS, Darstellung des gemeinen Deutschen und des Hamburgischen Handelsrechts für Juristen und Kaufleute, Bd. 3: Seerecht (Hamburg 1832). 
Hendrik M. PUNT, Het vennootschapsrecht van Holland. Het vennootschapsrecht van Holland, Zeeland en West-Friesland in de rechtspraak van de Hoge Raad van Holland, Zeeland en WestFriesland (Deventer 2010).

Joannes P. TAUNAY, Disputatio juridica inauguralis an et quousque exercitores navium ex magistrorum factis obligentur (Leiden 1802).

Oncko W. STAR NUMAN, Cornelis van Bynkershoek. Zijn leven en zijne geschriften (Leiden 1869).

Phillip J. THOMAs, Contributory Fault in Maritime Collisions in the Law of Holland, in: RIDA 48 (2001) 345-360.

Lambertus J. VAN APELDOORN (Hg.), Uit de practijk van het Hof van Holland in de tweede helft van de zestiende eeuw. Een handschrift (=OudVaderlandsche rechtsbronnen, $3^{\text {e }}$ reeks, VII, Utrecht 1938).

Cornelis VAN BIJNKERSHOEK, Observationes Tumultuariae, hg. von E. M. MeIjers u.a., 2 Bde (Haarlem 1926/1934).

DERS., Quaestiones juris privati (Leiden 1744).

Govaert C.J.J. VAN DEN BERGH, Der Präsident. Cornelis van Bijnkershoek, seine Bedeutung und sein Nachruhm, in: ZEuP 3 (1995) 423-437.

Dionysius G. VAN DER KEESSEL, Theses selectae juris Hollandici et Zelandici, ad supplendam Hugonis Grotii Introductionem ad jurisprudentiam Hollandicam (Amsterdam 1860).

DERS., Praelectiones iuris hodierni ad Hugonis Grotii Introductionem ad iurisprudentiam Hollandicam, ed. by P. VAN WARMELO u.a., V (Amsterdam/Kaapstad 1967).
Joannes VAN DER LINDEN, Regtsgeleerd, practicaal, en koopmans handboek (Amsterdam 1806).

DERS., 'Supplementum', in: Johannes VOET, Commentarius ad Pandectas, Bd. III (Utrecht 1793).

Simon VAN LeEUWEN, Het Rooms-Hollands regt (Amsterdam ${ }^{8} 1708$ ).

Johan P. VAN NIEKERK, The Development of the Principles of Insurance Law in the Netherlands from 1500 to 1800, Bd. II (Capetown 1998).

Cornelis VAN NIEUSTAD (NEOSTADIUS), Utriusque Hollandiae Zelandiae Frisiaeque curiae decisiones (The Hague 1667).

Adriaan P. VERWER, Nederlants see-rechten, avaryen en bodemeryen [...] (Amsterdam ${ }^{4} 1764$ ).

Johannes VOET, Commentarius ad Pandectas, Bd. I (Geneva 1778).

Hamilton VREELAND, Hugo Grotius: the Father of the Modern Science of International Law (New York 1917).

John WeSKeTT, A Complete Digest of the Theory, Laws, and Practice of Insurance (London 1781).

Jakob ZEYLEMAKER, Geschiedenis van de wetenschap van het burgerlijk procesrecht (praktijkrecht) in Nederland van de aanvang tot 1813 (=Geschiedenis der Nederlandsche rechtswetenschap. IV, I, Amsterdam 1952).

Reinhard ZIMMERMANN, Römisch-holländisches Recht. Ein Überblick, in: Robert FEENSTRA, Reinhard ZIMMERMANN (Hgg.), Das römischholländische Recht. Fortschritte des Zivilrechts im 17. und 18. Jahrhundert (Berlin 1992) 9-58. 